\title{
COUNTABLE ABELIAN GROUPS WITH A DISCRETE NORM ARE FREE
}

\author{
JOHN LAWRENCE ${ }^{1}$
}

\begin{abstract}
It is proved that a countable Abelian group with a norm which induces the discrete topology is free.
\end{abstract}

We will say that a function \|\| from an Abelian group $A$ to the real numbers $\mathbf{R}$ is a discrete norm if:

(1) \| $\|$ maps $A$ to the nonnegative real numbers;

(2) $\|a+b\| \leqslant\|a\|+\|b\|, \forall a, b \in G$;

(3) $\|n a\|=|n|\|a\|, \forall a \in G, \forall n \in \mathbf{Z}$;

(4) there is a positive number $r$ such that $\|a\| \geqslant r$ for all nonidentity elements $a$ of $A$.

If, in addition, $f$ maps to the nonnegative integers, then $f$ is said to be an integer-valued norm.

Lemma 1. If $A$ is an Abelian group with a norm, then $A$ is torsion-free.

Proof. If $0 \neq n \in \mathbf{Z}$ and $n a=0$, then $0=\|n a\|=|n|\|a\|$, so $\|a\|=0$, hence $a=0$.

Lemma 2. A discrete norm on the group $\mathbf{Z}^{n}=\mathbf{Z} \times \mathbf{Z} \times \cdots \times \mathbf{Z}$ ( $n$ times) induces a unique norm on $\mathbf{R}^{n}=\mathbf{R} \times \mathbf{R} \times \cdots \times \mathbf{R}$.

Proof. Let $\left(r_{1}, r_{2}, \ldots, r_{n}\right) \in \mathbf{Q}^{n}$ and let $k$ be a positive integer such that $k\left(r_{1}, r_{2}, \ldots, r_{n}\right) \in \mathbf{Z}^{n}$. Define \|\|$^{\prime}$ on $\mathbf{Q}^{n}$ by $\left\|\left(r_{1}, \ldots, r_{n}\right)\right\|^{\prime}=\frac{1}{k}\left\|\left(k r_{1}, k r_{2}, \ldots, k r_{n}\right)\right\|$. This is easily shown to be well defined and continuous, hence it may tend to $\mathbf{R}^{n}$ by continuity. That the norm is unique follows from the fact that our definition holds by necessity.

THEOREM 3. If $A$ is free Abelian, then there is an integer-valued norm on $A$.

Proof. Choose a Z-basis for $A$ and use the supremum norm.

TheOREM 4. Let $A$ be a countable Abelian group with a discrete norm. Then $A$ is a free Abelian group.

Proof. By a theorem of Pontryagin [1, p. 93], it is enough to show that every subgroup of finite rank is free. Since a discrete norm on $A$ induces a discrete norm

Received by the editors December 18, 1982 and, in revised form, May 25, 1983.

1980 Mathematics Subject Classification. Primary 20K99.

Key words and phrases. Abelian group, norm.

'Research partially supported by an NSERC Grant. 
on a subgroup, we may, without loss of generality, assume $A$ is of finite rank $n$. If $A$ is not free, then it is not finitely generated; hence, there is a proper ascending chain of free rank $n$ subgroups, $A_{1} \subseteq A_{2} \subseteq \cdots \subseteq A$, where the union of the chain is $A$. Suppose $A_{i}$ is generated as a free group of rank $n$ by the elements $\alpha_{i 1}, \alpha_{i 2}, \ldots, \alpha_{i n}$, that is, $A_{i}=\mathbf{Z} \boldsymbol{\alpha}_{i 1}+\mathbf{Z} \boldsymbol{\alpha}_{i 2}+\cdots+\mathbf{Z} \boldsymbol{\alpha}_{i n}$.

The embedding of $A_{i}$ into $A_{i+1}$ is represented by an $n \times n$ matrix

$$
M_{i}=\left(\begin{array}{cccc}
a_{11} & a_{12} & \cdots & a_{1 n} \\
a_{21} & a_{22} & \cdots & a_{2 n} \\
& \cdots & & \\
a_{n 1} & \cdots & & a_{n n}
\end{array}\right)
$$

where

$$
\alpha_{i+1 j}=a_{1 j} \alpha_{i 1}+a_{2 j} \alpha_{i 2}+\cdots+a_{n j} \alpha_{i n} .
$$

Since the embedding is proper, $\left|\operatorname{det} M_{i}\right|>1$. Since the matrix is integer valued, $\left|\operatorname{det} M_{i}\right| \geqslant 2$.

Suppose $N$ is a positive integer with the property that $\left\|\alpha_{1 j}\right\| \leqslant N$ for $j=1,2, \ldots, n$. Consider $A_{l}=\mathbf{Z} \alpha_{l 1}+\cdots+\mathbf{Z} \alpha_{l n} \cong \mathbf{Z}^{n} \subset \mathbf{R}^{n}$. The norm on $A$ induces one on $A_{l}$ which induces one on $\mathbf{R}^{n}$. Let $M$ be the matrix $M_{l-1} \cdot M_{l-2} \cdots M_{1}$ which maps $A_{1}$ into $A_{l}$. Then $\mid$ det $M \mid \geqslant 2^{l-1}$. Now the $n$-dimensional simplex generated by the vectors $\alpha_{11}, \alpha_{12}, \ldots, \alpha_{1 n}$ in $A_{l}$ has volume $C|\operatorname{det} M|$ where $C$ is a positive constant depending on $n$ (not on $l$ ). Then the $N$-ball in $\mathbf{R}^{n}$ coming from $A$, has volume $C \operatorname{det} M$, thus the 1-ball ( $=$ unit ball) has volume greater than or equal to $C / N^{n} \cdot 2^{l-1}$. Choose a positive integer $s$ such that for all nonidentity elements $x \in A$, we have $\|x\| \geqslant 1 / s$.

Now the $1 / 2 s$ ball has volume

$$
\geqslant\left(C / N^{n}(2 s)^{n}\right) 2^{l-1} \text {. }
$$

Choose $l$ large enough so that

$$
\left(C / N^{n}(2 s)^{n}\right) 2^{l-1}>2^{n} \text {. }
$$

By Minkowski's Theorem [2, p. 394], there is an element other than the identity element in $A$, which has norm less than $1 / 2 s$. This contradicts the fact that every nonidentity element has norm greater than or equal to $1 / s$. This contradiction proves the statement of the theorem.

THEOREM 5. Let $\Pi^{\infty} \mathbf{Z}$ be the countably infinite direct product of copies of $\mathbf{Z}$. There is no discrete norm on this group.

Proof. Let $e_{i}$ be the element with 1 in the $i$ th position and zeros elsewhere. Let $k_{1}=1$ and choose positive integers $k_{n}$ inductively so that

$$
r 2^{k_{n}}>\left(\sum_{i=1}^{n-1} 2^{k_{i}}\left\|e_{i}\right\|\right)+n
$$

Let $a=\left(2^{k_{1}}, 2^{k_{2}}, 2^{k_{3}}, \ldots\right)$ and choose $m$ so that $m>\|a\|$. Since

$$
a-\sum_{i=1}^{m-1} 2^{k_{i}} e_{i}=\left(0,0,0, \ldots, 2^{k_{m}}, 2^{k_{m+1}}, \ldots\right)
$$


we have

$$
\|a\|+\sum_{i=1}^{m-1} 2^{k^{\prime}}\|e\| \geqslant 2^{k^{m} r},
$$

a contradiction.

In [3] a function in two variables from a group to the integers is called a Yamabe function if it is bilinear. It is proved that a symmetric positive-definite Yamabe function on a group implies that the group is free Abelian. We offer a new proof of this in the case where the group is a countable Abelian group.

Theorem 6. If $A$ is a countable Abelian group with a positive-definite Yamabe function $Q$, then $A$ is free Abelian.

Proof. Since the function is a real inner product on $A$, there is an induced norm $|Q(x, x)|^{1 / 2}=\|x\|$. Since $Q(x, x)$ maps to the nonnegative integers, $\|x\| \geqslant 1$ if $x \neq 0$ in $A$. Thus $A$ is free Abelian by Theorem 4 .

Motivation and examples. The question of whether an Abelian group with an integer-valued norm is free appears to have been first asked by W. Ralph. In his thesis [4], Ralph studies a chain complex $A(X)$ for spaces $X$, where $A_{q}(X)=$ $\operatorname{span}_{\mathbf{z}}\left\{\tau: C(X) \rightarrow C\left(\Delta_{q}\right)\right\}$. Here $C(X)$ is the algebra of bounded continuous functions, $\Delta_{q}$ is the standard $q$-simplex, and $\tau$ ranges over algebra homomorphisms induced by continuous maps from $\Delta_{q}$ to $X$. There exists an integer-valued norm on the groups $A_{q}(X)$, where $X$ is a completely regular space. An affirmative answer to the question would greatly simplify the proofs of some theorems in Ralph's work (see Theorems 6.11 and 6.12).

Another example of an Abelian group equipped with an integer-valued norm is the Specker group. Given a set, the Specker group is the group of all bounded functions from the set to the integers. The norm in this case is the maximum of the absolute values of the range of the function. It should be noted that in this case the norm is not coming from a basis for the Abelian group. The freeness of the Specker groups for an arbitrary set was first proved by J. Nöbeling. A simpler proof was discovered by G. Bergman, however, the proof is still not easy (see [1] for details).

As we mentioned earlier, the existence of a Yamabe function on a group insures that it is free without any cardinality restriction. This gives some grounds for suspecting that an Abelian group with an integer-valued norm (or more generally a discrete norm) must be free.

\section{REFERENCES}

1. L. Fuchs, Infinite Abelian groups, Academic Press, New York, 1970.

2. G. Hardy and E. Wright, An introduction to the theory of numbers, Oxford Univ. Press, 1959.

3. B. H. Neumann and H. Neumann, On a class of Abelian groups, Arch. Math. 4 (1953), 79-85.

4. W. Ralph, Variants of the singular complex and their connections with Banach algebras, Čech cohomologv and co-products, Doctoral Thesis, Univ. of Waterloo, 1982.

Department of Pure Mathematics, University of Waterloo, Waterloo, Ontario, Canada N2L $3 \mathrm{Gl}$ 